\title{
Tuning controllers using the dual Youla parameterization
}

\author{
Niemann, Hans Henrik; Stoustrup, Jakob
}

Published in:

Proceedings of the American Control Conference

Link to article, DOI:

10.1109/ACC.2000.876718

Publication date:

2000

Document Version

Publisher's PDF, also known as Version of record

Link back to DTU Orbit

Citation (APA):

Niemann, H. H., \& Stoustrup, J. (2000). Tuning controllers using the dual Youla parameterization. In Proceedings of the American Control Conference (Vol. 2, pp. 1337-1338).

https://doi.org/10.1109/ACC.2000.876718

\section{General rights}

Copyright and moral rights for the publications made accessible in the public portal are retained by the authors and/or other copyright owners and it is a condition of accessing publications that users recognise and abide by the legal requirements associated with these rights.

- Users may download and print one copy of any publication from the public portal for the purpose of private study or research.

- You may not further distribute the material or use it for any profit-making activity or commercial gain

- You may freely distribute the URL identifying the publication in the public portal

If you believe that this document breaches copyright please contact us providing details, and we will remove access to the work immediately and investigate your claim. 


\section{Tuning Controllers using the Dual Youla Parameterization}

\author{
Henrik Niemann \\ Department of Automation \\ Technical University of Denmark \\ Building 326 \\ DK-2800 Lyngby, Denmark. \\ E-mail: hhn@iau.dtu.dk
}

\begin{abstract}
This paper describes the application of the Youla parameterization of all stabilizing controllers and the dual Youla parameterization of all systems stabilized by a given controller in connection with tuning of controllers. In the uncertain case, it is shown that the use of the Youla parameterization will allow us to optimize both the model and the controller.
\end{abstract}

\section{Introduction}

The Youla parameterization has been used in connection with both adaptive controllers (see e.g. [3]) as well as with gain scheduling controllers (see [2]). The main focus in this paper is to give a quantitative analysis of the use of parameterization in connection with tuning of controllers. It is shown that the use of the Youla parameterization can be applied also in this connection with several advantages. In the uncertain case, it will be shown that the Youla parameterization can both be applied in connection with optimization of the model as well as used in connection with controller tuning.

\section{Preliminary Results}

A system is given by:

$$
G(s)=\left[\begin{array}{lll}
G_{z w} & G_{z d} & G_{z u} \\
G_{e w} & G_{e d} & G_{e u} \\
G_{y w} & G_{y d} & G_{y u}
\end{array}\right]
$$

A coprime factorization of the system $G_{y u}(s)$ and a stabilizing controller $K(s)$ is given by:

$$
\begin{array}{ll}
G_{y u}=N M^{-1}=\tilde{M}^{-1} \tilde{N}, \quad N, M, \tilde{N}, \tilde{M} \in \mathcal{R} \mathcal{H}_{\infty} \\
K \quad=U V^{-1}=\tilde{V}^{-1} \tilde{U}, \quad U, V, \tilde{U}, \tilde{V} \in \mathcal{R} \mathcal{H}_{\infty}
\end{array}
$$

where the eight matrices must satisfy the double Bezout equation, see $[3,4]$.

All controllers that stabilize $G_{y u}$ is given by:

$$
K(Q)=\mathcal{F}_{l}\left(\left[\begin{array}{cc}
U V^{-1} & \tilde{V}^{-1} \\
V^{-1} & -V^{-1} N
\end{array}\right], Q\right)
$$

\author{
Jakob Stoustrup \\ Dept. of Control Engineering \\ Aalborg University \\ Fr. Bajersvej 7C \\ DK-9220 Aalborg $\varnothing$, Denmark \\ E-mail: jakob@control.auc.dk \\ URL: www.control.auc.dk/ jakob
}

where $Q \in \mathcal{R} \mathcal{H}_{\infty}$. All systems stabilized by $K(s)$ is given by:

$$
G(S)=\mathcal{F}_{l}\left(\left[\begin{array}{cc}
N M^{-1} & \tilde{M}^{-1} \\
M^{-1} & -M^{-1} U
\end{array}\right], S\right)
$$

where $S \in \mathcal{R} \mathcal{H}_{\infty}$.

The connection between the real system described by $G_{y u}(\Delta)=G_{y u}+G_{y w} \Delta\left(I-G_{z w} \Delta\right)^{-1} G_{z u}$ and the $S$ parameter is given by, [1]:

$$
S(\Delta)=T_{3} \Delta\left(I-T_{1} \Delta\right)^{-1} T_{2}
$$

where $T_{1}=G_{z w}+G_{z u} U \tilde{M} G_{y w}, T_{2}=G_{z u} M$, and $T_{3}=$ $\tilde{M} G_{y w}$.

The above description of change of a system is based on the $\Delta$ formulation. A special case of the above general description is when the system is changed from $G_{y u, 0}$ to $G_{y u, 1}$. Using the coprime factorization of both $G_{y u, 0}$ and $G_{y u, 1}$, the dual Youla parameter $S$ is then given by

$$
S=\tilde{N}_{0} M_{1}-\tilde{M}_{0} N_{1}
$$

\section{Main Results}

Let us consider uncertain systems. When the system includes uncertain blocks $\Delta$, it is not directly possible to describe the system from an input/output point of view by using the dual Youla parameterization. It is only possible to use the dual Youla parameterization to describe the system from the control input $u$ to the measurement output $y$. As a result of this, it is not directly possible to use the dual Youla parameter in connection with optimization of the performance. It is possible to minimize the performance transfer function $T_{e d}$ directly, if the input and output signals are linear combinations of $u$ and $y$.

Now, let us use the Youla parameterization of the controllers to change the controller. By introducing $Q=\sum_{i=1}^{p} \alpha_{i} Q_{i}$ and using $K(Q), T_{1}(\alpha)$ is given by

$$
T_{1}(\alpha)=G_{z w}+G_{z u} \sum_{i=0}^{p} \alpha_{i} U_{i} \tilde{M} G_{y w}
$$

$0-7803-5519-9 / 00 \$ 10.00 \odot 2000$ AACC 
The vector $\alpha$ is a set of controller scheduling parameters. $S(\Delta, Q)$ is then given by

$$
S(\Delta, Q)=\tilde{M} G_{y w} \Delta\left(I-T_{1}(\alpha) \Delta\right)^{-1} G_{z u} M
$$

In this case where both the system and the controller can/will change, the design problem turns out to be more complicated. However, when a more realistic description of the system is included in the problem formulation, it will be possible to do other things in connection with the optimization of the controller. It is quite clear that if the model of the system and the real system is identical, then we get directly that

$$
S(\Delta)=0
$$

This strongly indicates that trying to make $S$ small often will be a desirable objective. Minimizing $S$ by updating the model, we will get a more precise model for the applied operating point. This needs to be followed by an update of the controller such that the performance is optimized. The most natural way to do this optimization of the controller is to apply a gain scheduling controller. Minimizing $S$ by tuning the controller, the effect from the uncertainties is minimized, i.e. $Q$ is optimized with respect to $S(\Delta)$. In this case, the performance from the nominal case will be recovered. A short description of the two cases follows below.

\subsection{Optimization of the model}

Let us assume that the real system can be described as a combination of a number of models. In [2], an LFT description of the system given by

$$
G_{y u}(\theta)(t)=\mathcal{F}_{u}\left(G_{p a r}, \theta(t)\right)
$$

where $\theta(t)$ is a vector of time varying parameters and $G_{p a r}$ is a known part of the system.

There exist a number of methods for estimation of the parameter vector $\theta$. In our case, it is possible to validate the identified parameter vector $\hat{\theta}(t)$, because the parameter vector consists of real parameters. The validation of $\hat{\theta}(t)$ can be done as follows.

Based on the identified parameter vector $\hat{\theta}(t)$, the real parameter is given by:

$$
\tilde{\theta}(t)=\theta_{0}+\hat{\theta}(t)
$$

where $\theta_{0}$ is the parameter vector for the nominal system. The model of the system can now be updated. Based on this updated model, a new dual Youla parameter $S(\tilde{\theta}(t))$ can be calculated by:

$$
\begin{aligned}
S(\tilde{\theta}(t))= & V^{-1}\left(I-G_{y u}(\theta(t)) U V^{-1}\right)^{-1} \\
& \left(G_{y u}(\theta(t)) M(\tilde{\theta}(t))-N(\tilde{\theta}(t))\right)
\end{aligned}
$$

As a direct consequence of this equation, the identified parameter vector $\tilde{\theta}(t)$ is the optimal vector, i.e. $\tilde{\theta}(t)=$ $\theta(t)$, if and only if

$$
S(\tilde{\theta}(t))=0
$$

If the identified model given by $G_{y u}(\tilde{\theta}(t))$ does not describe the real system well enough, i.e. the dual Youla parameter $S(\tilde{\theta}(t))$ is too large in some sense, a new identification of $\theta$ can be derived based on the new model of the system given by $G_{y u}(\tilde{\theta}(t))$.

Based on the estimated parameter vector $\tilde{\theta}$, the controller can now be updated/redesigned. The gain scheduling method applied in connection with estimation of the parameter vector $\theta$ has been considered in [2].

\subsection{Optimization of the controller}

An alternative to the above scheme is to optimize the controller directly such that the dual Youla parameter $S$ is minimized. This can be done in a number of different ways. In [3], both off-line as well as on-line methods have been considered in connection with minimization of $S$. These methods are based on a least squares minimization.

As an alternative to this least squares minimization, an $\mathcal{H}_{\infty}$ optimization can be applied instead. To that end, observe, that evaluating the worst case $\mathcal{H}_{\infty}$ norm of (6) is a $\mu$-analysis problem. Further, we note that the $\Delta$ feedback path $T_{1}(\alpha)=G_{z w}+G_{z u} \sum_{i=0}^{p} \alpha_{i} U_{i} \tilde{M} G_{y w}$ can be written in the form

$$
T_{1}(\alpha)=G_{z w}+G_{z u} \bar{\alpha}\left(\begin{array}{c}
U_{0} \\
U_{1} \\
\vdots \\
U_{p}
\end{array}\right) \tilde{M} G_{y w}
$$

which is a static output feedback form in the unknown matrix $\bar{\alpha}=\left(\begin{array}{llll}\alpha_{0} & \alpha_{1} & \ldots & \alpha_{p}\end{array}\right)$.

Hence, the associated optimization problem involves in principle finding a static solution to a $\mu$-problem. This problem is highly infeasible from a numerical point of view, but a suboptimal solution can be found by using LMI's. The static constraint can then be dealt with by using alternating projections on a number of convex sets and a single nonconvex set corresponding to a rank constraint which has been reported feasible in the literature.

\section{References}

[1] H.H. Niemann. Application of the dual Youla parameterization. In European Control Conference, ECC'99, Karlsruhe, Germany, 1999.

[2] H.H. Niemann and J. Stoustrup. Gain scheduling using the Youla parameterization. In Proceedings of the 38th IEEE Conference on Decision and Control, pages 2306-2311, Phoenix, AZ, USA, 1999.

[3] T.T. Tay, I.M.Y. Mareels, and J.B. Moore. High performance control. Birkhäuser, 1997.

[4] K. Zhou, J.C. Doyle, and K. Glover. Robust and optimal control. Prentice Hall, 1996. 